\title{
Acute type A aortic dissection mimicking a congenital supravalvular aortic membrane
}

Ioannis Dimarakis, MRCS, ${ }^{a}$ Rashmi Yadav, FRCS, ${ }^{a}$ Sandeep Bahia, MBBS, ${ }^{\text {a }}$ Christoph Juli, FRCR, ${ }^{\text {b }}$ Nicola Strickland, FRCP, FRCR, ${ }^{b}$ Raffi Kaprielian, MRCP, ${ }^{c}$ and Jonathan Anderson, FRCS(CTh), ${ }^{\text {a }}$ London, United Kingdom

Supplemental material is available online.

Video clip is available online.
From the Departments of Cardiothoracic Surgery, ${ }^{\mathrm{a}}$ Imaging, ${ }^{\mathrm{b}}$ and Cardiology, ${ }^{\mathrm{c}} \mathrm{Ham}-$ mersmith Hospital, London, United Kingdom.

Received for publication May 28, 2007; accepted for publication June 21, 2007.

Address for reprints: Ioannis Dimarakis, MRCS, Department of Cardiothoracic Surgery, Imperial College London, Hammersmith Hospital Campus, Du Cane Rd, London W12 ONN, United Kingdom (E-mail: ioannis.dimarakis@imperial.ac.uk).

J Thorac Cardiovasc Surg 2007;134:1340-1

$0022-5223 / \$ 32.00$

Copyright $\odot 2007$ by The American Association for Thoracic Surgery

doi:10.1016/j.jtcvs.2007.06.029

\section{Clinical Summary}

A 45-year-old construction worker presented to his local casualty department with a 3-week history of recurrent epigastric pain associated with shortness of breath on exertion. He was otherwise a healthy nonsmoker with no significant medical history. Clinical examination revealed tachycardia with pansystolic and diastolic decrescendo murmurs best heard at the lower left sternal border. A routine chest radiograph showed no abnormalities, and routine laboratory investigations showed mildly elevated levels of transaminases and C-reactive protein. Results of viral hepatitis screen and serial blood cultures were negative. Transthoracic and transesophageal echocardiograms suggested a membrane $2 \mathrm{~cm}$ above the aortic valve annulus within an aneurysm of the proximal ascending thoracic aorta (diameter $5 \mathrm{~cm}$ ). Significant 4-chamber dilatation with impairment of left ventricular systolic function was noted; in addition, severe acute aortic regurgitation, mild functional mitral regurgitation, and pulmonary hypertension were recorded. A gadolinium-enhanced cardiac magnetic resonance (CMR) study showed a circumferential membrane arising at a $90^{\circ}$ angle from the wall of the aortic root (Figure 1, A). Aortic flow measured on CMR demonstrated a minimal opening area of $4.15 \mathrm{~cm}^{2}$ within this membrane situated directly above the normal-sized opening of the tricuspid aortic valve. No other aortic wall abnormality within the thoracic aorta suggestive of a dissection was seen. A CMR cine clip is included in the coronal plane through the aortic outflow tract and left ventricle, showing an apparent supravalvular aortic membrane approximately $2 \mathrm{~cm}$ above a moderately regurgitant aortic valve (see Video).

At operation, a well-defined demarcation was noted on a dilated proximal ascending aorta (Figure E1, A). After aortotomy, a circumferential intimal tear was identified; the intimal flap remained anchored distally, explaining the absence of a false lumen and the radiologic appearances of a supravalvular membrane (Figure 2, $A$ and $B$ ). Aortic root replacement with the modified Bentall technique was performed (Figure E1, $B$ ); the patient had an uncomplicated postoperative course.

\section{Discussion}

Described hallmarks of dissection include the presence of an intimal membrane or flap dividing a true and a false lumen, entry and re-entry tears, periaortic hematoma, pericardial effusion or tamponade, and aortic regurgitation in dissection of the ascending aorta with aortic valve involvement. ${ }^{1}$ A wide range of aortic pathologic conditions besides aortic dissection may clinically underlie acute aortic syndrome. These include a variety of disorders lacking the characteristic mobile intimal flap-such as intramural hematoma, penetrating aortic ulcer, and localized intimal tear without dissection - that may prove an imaging diagnostic challenge. ${ }^{2}$ Intimal tears in type A aortic dissections are usually transverse, with more than half the aortic circumference being spared. Approximately 20 cases of circumferential tear of the intima, presenting with either antegrade or retrograde intussusception, have been reported to date. ${ }^{3}$ In our case, all imaging modalities implied the possible presence of a perforated membranous type supravalvular aortic stenosis ${ }^{4}$ leading to enlargement of the aortic root and severe aortic regurgitation. A definitive diagnosis was made only intraoperatively. 


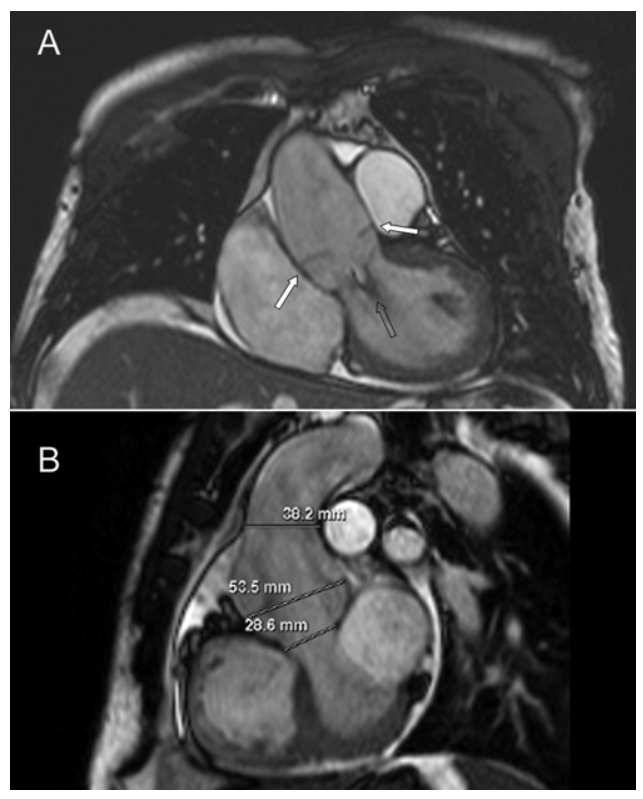

Figure 1. Cardiac magnetic resonance images. A, Coronal image through the aortic outflow tract showing a circumferential membrane (filled arrows) seen to arise at a $90^{\circ}$ angle from the wall of the aortic root $2.1 \mathrm{~cm}$ distal to the aortic valve. The very mobile medial part of this membrane was obstructing blood flow directly superior to the aortic valve (open arrow). B, Aortic root, with maximum dilatation of $5.4 \mathrm{~cm}$.

\section{References}

1. Flachskampf FA. Assessment of aortic dissection and hematoma. Semin Cardiothorac Vasc Anesth. 2006;10:83-8.

2. Beauchesne LM, Veinot JP, Brais MP, Burwash IG, Chan KL. Acute aortic intimal tear without a mobile flap mimicking an intramural hematoma. J Am Soc Echocardiogr. 2003;16:285-8.

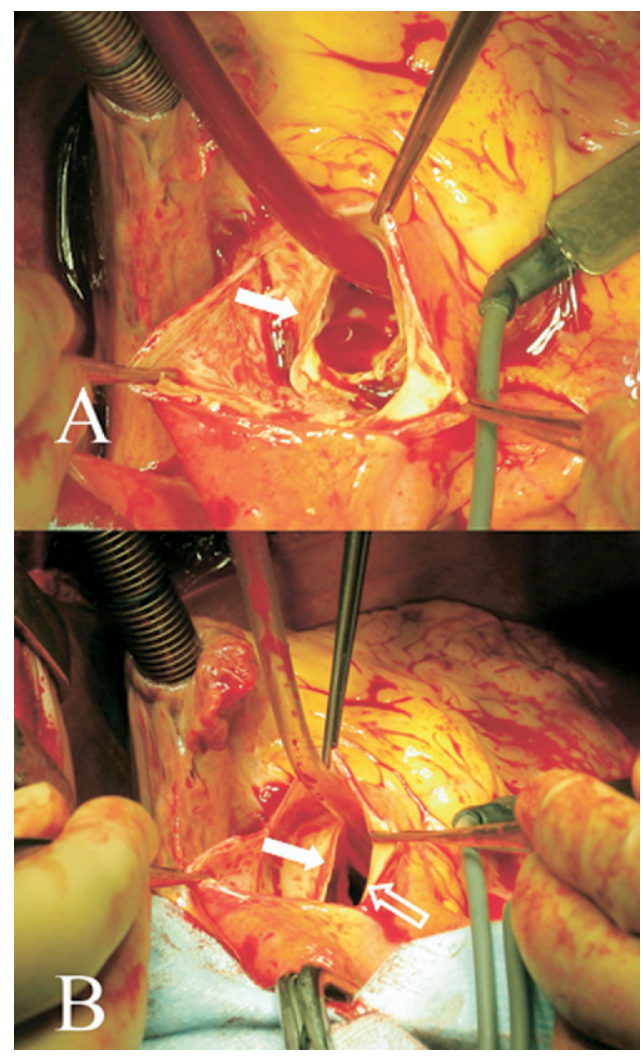

Figure 2. Intraoperative photographs. A, View of the circumferential intimal tear (filled arrows) after aortotomy. B, The tricuspid aortic valve may be seen below the tear (open arrow).

3. Shah PJ, Singh SS, Chaloob SS, Lang C, Taylor J, Edwards JR. Intimo-intimal intussusception of the aorta. Ann Thorac Surg. 2006;82: 2274-6.

4. Covington MK, Byrd IBF. Congenital heart disease in adults: echocardiographic evaluation of left and right ventricular outflow tract obstruction. Prog Pediatr Cardiol. 2003;17:9-19. 


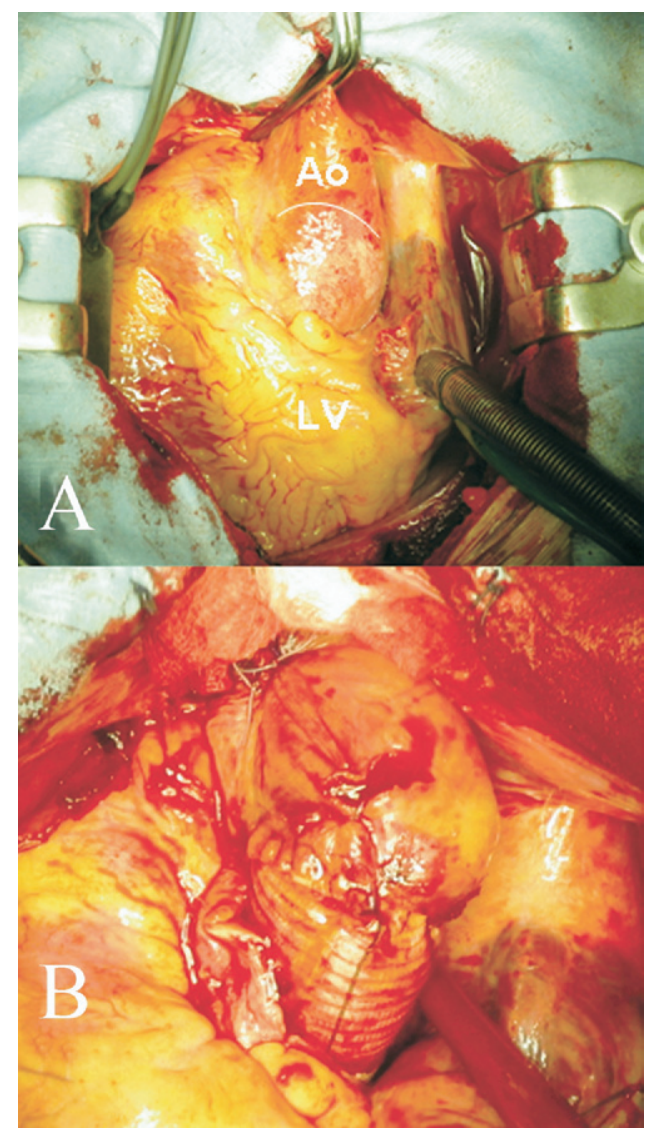

Figure E1. Intraoperative photographs. A, Aneurysmatic dilatation of the proximal ascending aorta (Ao); clear demarcation may be seen between affected and nonaffected aorta (white line). B, The size 25 Carbo-Seal composite graft (CarboMedics, Austin, Tex) in position. LV, Left ventricle. 